\title{
Using economic evaluations to support acupuncture reimbursement decisions: current evidence and gaps
}

\author{
Hongchao Li and colleagues explore the global challenges of including economic evaluations in \\ decisions about reimbursement for acupuncture
}

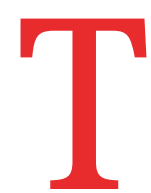

he process of making decisions about healthcare reimbursement is inherently complex. In addition to effectiveness and safety, social, economic, political, geographical, and institutional non-medical factors often are important in decision making.

In past decades, the process of making decisions about reimbursement has evolved, increasingly incorporating economic evaluation. In China, the United Kingdom, Australia, and Canada, it has become one of the most critical factors determining whether a drug or a health technology can enter the reimbursement list.

Clinicians in over 183 countries, motivated by increasing evidence of effectiveness, ${ }^{1-5}$ use acupuncture, a non-pharmaceutical intervention, predominantly for pain relief but also in a wide variety of other conditions. ${ }^{6}$ Although private health insurance often covers acupuncture, public health insurance rarely does. ${ }^{78}$ Given the high prevalence of chronic pain, ${ }^{910}$ extensive use of

\section{REY MESSAGES}

- Economic evaluations of acupuncture rarely consider contextual factors or long term outcomes, and their reporting quality is poor

- In the UK, Germany, US, China, and Switzerland, economic evaluation has a limited role in reimbursement decisions

- Both the quantity and quality of current economic evaluations of acupuncture interventions are insufficient to meet the needs of health decision makers

- Adequate support for decision making about acupuncture will require additional high quality economic evaluations carried out in a wider variety of jurisdictions acupuncture can use considerable health resources. ${ }^{11}$ Thus like other interventions, decisions about reimbursement for acupuncture should ideally include economic evaluation.

We summarise the current status of evidence for the economic evaluation of acupuncture, provide examples of the extent to which evidence of its cost effectiveness is used in decision making about reimbursement, raise challenges of applying the evidence to decisions about reimbursement, and provide corresponding suggestions.

\section{Problems with economic evaluations} Lack of contextual factors and long term outcomes

Most of the published economic evaluations of acupuncture are based on trials ${ }^{12-14}$; therefore, the trial design inevitably affects its quality. Few randomised controlled trials have measured contextual factors and long term outcomes, thus limiting the credibility of associated economic evaluations. Contextual factors, including practitioner expertise, setting, patients' previous experiences, and social position, as well as co-interventions, can affect the model for evaluation of cost effectiveness.

Because economic models for management of chronic pain often need to simulate at least 5 years or even a lifetime, ${ }^{15-17}$ measurement of long term outcomes is crucial. Recently, published systematic reviews show that the longest follow-up of existing randomised controlled trials of acupuncture for chronic pain is 13 months. ${ }^{1819}$ The only long term simulation model (that is, the Markov model) using outcomes from three 3-12 month trials simulated five year chronic pain relief and analysed the cost effectiveness of acupuncture for chronic pain. ${ }^{15}$ In such a situation, the use of transition probabilities derived from short term clinical trials to extrapolate to long term outcomes in an economic model might overestimate or underestimate the incremental cost effectiveness ratio. Thus the absence of long term outcome measures in acupuncture studies further undermines the credibility of associated economic analyses. ${ }^{16}$

\section{Poor reporting quality}

Poor reporting quality limits the usefulness of economic evidence in informing reimbursement decisions. ${ }^{12-14}$ Problems include poor reporting of the following: (a) intervention and comparator; (b) patients' characteristics; (c) productivity outcomes (eg, time lost from work); (d) definitions of primary outcome measure(s); (e) separate documentation of quantities of resource use and their unit costs; and (f) justification for sensitivity analysis and range of variables.

\section{Limited applicability}

To support reimbursement decision making, the National Institute for Health and Care Excellence (NICE) suggests evaluating the applicability-that is, the relevance to a specific reimbursement question-of economic evaluations. Health system, costs, clinical practice, and health preference sources-that is, in which population researchers elicit health utility scores-are the main determinants for the applicability of economic evaluations. For example, when considering the cost of acupuncture for a healthcare system, particularly if tax funded, the evaluation should consider space, equipment, practitioners' time, training, and their effect on other health provisions.

Most of the existing economic evaluations of acupuncture have been conducted in Germany, ${ }^{12-1420}$ the UK, ${ }^{12-1421-23}$ and the US, ${ }^{24-26}$ each of which has its unique health system, costs, clinical practice, and population health preferences. Therefore, it is difficult to generalise the results to other countries.

For example, for the health preference measures, analysis of the cost benefit of acupuncture most commonly used the EQ-5D (European quality-of-life five 
dimension instrument) and SF-6D (Short Form 6 Dimensions). ${ }^{12-14}$ Value sets of EQ-5D and SF-6D for Germany, the UK, and the US do not necessarily reflect other countries' population health preferences. Moreover, as clinical practice and health technology costs change over time, previously published economic evaluations might not provide valuable information for current decisions about reimbursement decision.

Given the paucity of evaluations outside Germany, the UK, and the US, decision makers in other countries must rely on studies published elsewhere, thus limiting applicability to their setting. Unlike innovative drugs, the industry rarely supports economic evaluations for acupuncture. Solving this problem of evaluations specific to particular jurisdictions will probably require government funding of research.

\section{Limited use of economic evaluations in reimbursement decisions}

Health systems and their process for making decisions about reimbursement vary widely across countries. In countries in which public health insurance reimburses acupuncture treatment, the types of evidence (eg, on effectiveness, safety, and cost) considered in decision making differ. To explore the extent of the use of economic evaluation in reimbursement decision making, we will comment on the situation in five countries.

In China, the Beijing Municipal Medical Insurance Bureau, based on expert opinion and a list of interventions prohibited from being reimbursed, decided in around 2000 to reimburse acupuncture for all conditions. ${ }^{27}$ This decision process did not use economic evaluation evidence. China's decision making process for access to basic medical insurance has, however, undergone immense changes in recent years. In negotiations about access to the national reimbursement drug list, China National Healthcare Security Administration requires a cost effectiveness analysis and budget impact analysis. ${ }^{28}$ We expect that, in the future, economic evaluation will also inform reimbursement decisions for non-pharmaceutical interventions, including acupuncture.

In Switzerland, after a public vote in 2009 , complementary medicine is now mentioned in the Swiss constitution Article 118a-"Within the scope of their responsibilities, the confederation and the cantons shall ensure that complementary medicine is comprehensively taken into account." Given that Switzerland has little local economic evaluation evidence, the reimbursement of acupuncture in the basic health insurance system is based on evidence of effectiveness and political considerations, which covers all conditions.

The UK's NHS provides free public healthcare to all British residents. NICE, the primary role of which is to improve outcomes for people using NHS services, has a long history of using Health Technology Assessment (HTA) to support evidence based decision making. A full HTA usually assesses efficacy, safety, cost effectiveness, and ethics.

NICE has conducted an HTA of acupuncture for chronic pain, comparing the cost effectiveness of acupuncture with usual care from the NHS perspective. Using a systematic review of clinical evidence, the review group constructed a lifetime economic evaluation model. Based on the HTA results (including that acupuncture is cost effective for chronic pain), a NICE clinical practice guideline recommends acupuncture for chronic pain in a community setting. ${ }^{16}$ However, access to free acupuncture services depends on the policy of the local clinical commissioning group, and patients can get limited courses of treatment only if recommended by their general practitioner. ${ }^{29}$ Only a few NHS services (eg, Royal London Hospital for Integrative Medicine) provide free acupuncture.

In Germany, public insurance covers acupuncture only for low back pain and knee pain. Two large, three arm, randomised controlled trials, carried out between 1999 and 2005, including usual care, sham acupuncture, and true acupuncture, informed these decisions. ${ }^{3031}$ Although several studies showed that acupuncture was cost effective within the accepted thresholds for low back pain ${ }^{32} 33$ and osteoarthritis, ${ }^{34}$ decisions about the reimbursement did not fully consider this evidence. Health insurance reimbursement decisions did not require economic evaluations at this time. ${ }^{35}$

In the US, private health insurance and government health insurance coexist. The Centers for Medicare and Medicaid Services make decisions about reimbursement. The extent of evidence informed decisions has increased. For instance, the reimbursement decision for low back pain considered clinical trial evidence that acupuncture in comparison with no intervention improves pain and function moderately and is supported by moderate certainty evidence. Compared with medication (non-steroidal antiinflammatory drugs, muscle relaxants, or analgesics), acupuncture shows small but important improvement (supported by low certainty evidence). Most of the relevant trials included younger patients, and results therefore provide only indirect evidence for patients over $60 .^{13637}$ To inform decisions about reimbursement further, the National Institutes of Health therefore funded a large randomised trial in patients over 60 , identifying the providers, assessing the cost effectiveness of acupuncture, and the optimal number of treatment sessions. While waiting for the trial results, hoping to avoid the harm caused by opioid misuse, in January 2020 , the Centers for Medicare and Medicaid Services issued a decision memorandum covering acupuncture for chronic low back pain. ${ }^{38}$ This decision considered the effectiveness and safety of acupuncture but did not use economic evaluation evidence.

The experience in these five countries shows that economic evaluation has a limited role in current decisions about reimbursement for acupuncture. Although using economic evaluation can help to optimise the allocation of health resources when making decisions about reimbursement for acupuncture, political and other factors often play more important roles than the evidence.

\section{Suggestions}

To deal with the challenges described, we have several suggestions for different stakeholders. For reimbursement decision makers in every health system, we suggest increasing use of economic evaluation. Governments and non-profit HTA agencies should provide more funding and organisational support to conduct local acupuncture economic evaluations.

Researchers conducting acupuncture economic evaluations or HTA should improve reporting quality by following commonly used quality checklistsfor example, the BMJ checklist ${ }^{39}$ or the Consolidated Health Economic Evaluation Reporting Standards (CHEERS). ${ }^{40}$

Health economists and clinicians who offer acupuncture should work collaboratively to design high quality economic evaluations, consider contextual factors, including the qualifications of acupuncture practitioners; measure long term outcomes; conduct analysis; and interpret results appropriately. Such collaboration can help to provide better measurement of the efficacy of acupuncture and more credible economic evaluations. 


\section{Conclusion}

Although optimal resource allocation requires reimbursement decisions about acupuncture to consider economic evaluation evidence, such evidence has little role in health decision making, including in the five countries we have particularly mentioned. The challenges of using economic evaluation evidence in reimbursement decisions for acupuncture include (a) infrequent consideration of contextual factors; (b) infrequent measurement of long term outcomes; (c) poor reporting quality; (d) limitation of economic evaluation to a small number of countries and settings; (e) limitations in decision making that rarely considers the evidence for economic evaluation; and (f) limited resources to carry out economic evaluations. To overcome these challenges, decision makers should increasingly use economic evaluation evidence and allocate funding to generate evidence for reimbursement. To increase the credibility of economic models, researchers need to measure both contextual factors and long term outcomes, improve reporting quality, and increase collaboration with clinician providers of acupuncture.

Contributors and sources: $\mathrm{HL}$ and $\mathrm{XuJ}$ conceived the idea and drafted the manuscript; PMH and CMW drafted the US, Germany, and Switzerland examples; WG, XiJ, PS, and LY provided the Chinese example; and LH, Y-QZ, DO, GG, YZ, and YC revised the manuscript critically for important intellectual content. $\mathrm{HL}$ and $\mathrm{XuJ}$ contributed equally as first authors. LH and Y-QZ contributed equally and are the guarantors.

Competing interests: We have read and understood BMJ policy on declaration of interests, and we have no conflicts of interest to declare.

Provenance and peer review: Commissioned; externally peer reviewed.

This article is part of a collection funded by the special purpose funds for the belt and road, China Academy of Chinese Medical Sciences, National Natural Science Foundation of China, the National Center for Complementary and Integrative Health, the Innovation Team and Talents Cultivation Program of the National Administration of Traditional Chinese Medicine, the Special Project of "Lingnan Modernization of Traditional Chinese Medicine" of the 2019 Guangdong Key Research and Development Program, and the Project of First Class Universities and High-level Dual Discipline for Guangzhou University of Chinese Medicine. The BMJ commissioned, peer reviewed, edited, and made the decision to publish. Kamran Abbasi was the lead editor for The BMJ. Yu-qing Zhang advised on commissioning for the collection, designed the topic of the series, and coordinated the author teams. Gordon Guyatt provided valuable advice and guidance.

Hongchao Li, associate professor ${ }^{1,2}$

Xuejing Jin, professor ${ }^{3}$

Patricia M Herman, senior scientist ${ }^{4}$

Claudia M Witt, professor ${ }^{5}$

Yingyao Chen, professor $^{6,7}$
Weijuan Gang, professor ${ }^{8,9}$

Xianghong Jing, professor ${ }^{8,9}$

Ping Song, professor ${ }^{10}$

Longhui Yang, professor $^{10}$

Dan Ollendorf, director of value measurement and global health initiatives ${ }^{11}$

Yuan Zhang, assistant professor ${ }^{12}$

Gordon Guyatt, professor ${ }^{12,13}$

Luqi Huang, professor ${ }^{9,10}$

Yu-Qing Zhang, executive director ${ }^{8,12,14,15}$

${ }^{1}$ School of International Pharmaceutical Business, China

Pharmaceutical University, Nanjing, China

${ }^{2}$ Centre for Pharmacoeconomics and Outcomes Research, China Pharmaceutical University, Nanjing, China

${ }^{3}$ Centre for Evidence-based Chinese Medicine, Beijing

University of Chinese Medicine, Beijing, China

${ }^{4}$ RAND Corporation, USA

${ }^{5}$ Institute for Complementary and Integrative Medicine, University Hospital Zurich and University of Zurich, Zurich, Switzerland

${ }^{6}$ School of Public Health, Fudan University, Shanghai, China

${ }^{7}$ National Health Commission Key Laboratory of Health Technology Assessment, Fudan University, Shanghai, China

${ }^{8}$ Institute of Acupuncture and Moxibustion, China Academy of Chinese Medical Sciences, Beijing, China

${ }^{9}$ China Centre for Evidence Based Traditional Chinese Medicine, China Academy of Chinese Medical Sciences, Beijing, China

${ }^{10}$ China Academy of Chinese Medical Sciences, Beijing, China

${ }^{11}$ Center for the Evaluation of Value and Risk in Health, Institute for Clinical Research and Health Policy Studies, Tufts Medical Center, USA

${ }^{12}$ Department of Health Research Methods, Evidence, and Impact, McMaster University, Hamilton, Ontario, Canada

${ }^{13}$ Department of Medicine, Faculty of Health Sciences, McMaster University, Hamilton, ON, Canada

${ }^{14}$ Centre for Evidence Based Integrative Medicine-Clarity Collaboration, Guang'anmen Hospital, China Academy of Chinese Medical Sciences, Beijing, China

${ }^{15}$ Ningbo Nottingham Grade Centre, University of Nottingham, Ningbo, China

Correspondence to: L Huang

huangluqi01@126.com

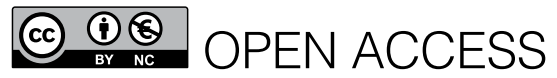

This is an Open Access article distributed in accordance with the Creative Commons Attribution Non Commercial (CC BY-NC 4.0) license, which permits others to distribute, remix, adapt, build upon this work non-commercially, and license their derivative works on different terms, provided the original work is properly cited and the use is non-commercial. See http://creativecommons.org/licenses/by-nc/4.0/.

\section{Check for updates}

1 Vickers AJ, Vertosick EA, Lewith GA, et al. cupuncture Trialists' Collaboration. Acupuncture for chronic pain: update of an individual patient data metaanalysis. J Pain 2018;19:455-74. doi:10.1016/j. jpain.2017.11.005

2 Tedesco D, Gori D, Desai KR, et al. Drug-free interventions to reduce pain or opioid consumption after total knee arthroplasty: a systematic review and meta-analysis. JAMA Surg 2017;152:e172872.

doi:10.1001/jamasurg.2017.2872

3 Mao JJ, Liou KT, Baser RE, et al. Effectiveness of electroacupuncture or auricular acupuncture vs usual care for chronic musculoskeletal pain among cancer survivors: the PEACE randomized clinical trial. JAMA Oncol 2021;7:720-7. doi:10.1001/ jamaoncol.2021.0310

4 Xu S, Yu L, Luo X, et al. Manual acupuncture versus sham acupuncture and usual care for prophylaxis of episodic migraine without aura: multicentre, randomised clinical trial. BMJ 2020;368:m697. doi:10.1136/bmj.m697

5 Deng ZQ, Zheng H, Zhao L, Zhou SY, Li Y, Liang FR. Health economic evaluation of acupuncture along meridians for treating migraine in China: results from a randomized controlled trial. BMC Complement Altern Med 2012;12:75. doi:10.1186/1472-6882-12-75

6 World Health Organization. WHO traditional medicine strategy: 2014-2023. 2013. https://www.who.int/ publications/i/item/9789241506096

7 Bleck R, Marquez E, Gold MA, Westhoff CL. A scoping review of acupuncture insurance coverage in the United States. Acupunct Med 2021;39:461-70.

8 Institute for Clinical and Economic Review. Cognitive and mind-body therapies for chronic low back and neck pain: effectiveness and value. 2017. http://icerorg.wpengine.com/wp-content/ uploads/2020/10/CTAF_LBNP_Final_Evidence_ Report_110617.pdf

9 Fayaz A, Croft P, Langford RM, Donaldson LJ, Jones GT. Prevalence of chronic pain in the UK: a systematic review and meta-analysis of population studies. BMJ Open 2016;6:e010364. doi:10.1136/ bmjopen-2015-010364

10 Sá KN, Moreira L, Baptista AF, et al. Prevalence of chronic pain in developing countries: systematic review and meta-analysis. Pain Rep 2019;4:e779. doi:10.1097/PR9.0000000000000779

11 Phillips CJ. Economic burden of chronic pain. Expert Rev Pharmacoecon Outcomes Res 2006;6:591-601. doi:10.1586/14737167.6.5.591

12 Herman PM, Poindexter BL, Witt CM, Eisenberg DM. Are complementary therapies and integrative care cost-effective? A systematic review of economic evaluations. BMJ Open 2012;2:e001046. doi:10.1136/bmjopen-2012-001046

13 Kim SY, Lee H, Chae Y, Park HJ, Lee H. A systematic review of cost-effectiveness analyses alongside randomised controlled trials of acupuncture. Acupunct Med 2012;30:273-85. doi:10.1136/ acupmed-2012-010178

14 Ambrósio EM, Bloor K, MacPherson H. Costs and consequences of acupuncture as a treatment for chronic pain: a systematic review of economic evaluations conducted alongside randomised controlled trials. Complement Ther Med 2012;20:364-74. doi:10.1016/j. ctim.2012.05.002

$15 \operatorname{Kim} \mathrm{N}$, Yang B, Lee T, Kwon S. An economic analysis of usual care and acupuncture collaborative treatment on chronic low back pain: a Markov model decision analysis. BMC Complement Altern Med 2010;10:74. doi:10.1186/1472-6882-10-74

16 National Institute for Health and Care Excellence. Chronic pain (primary and secondary) in over 16s: assessment of all chronic pain and management of chronic primary pain. 2021. https://www.nice.org.uk/ guidance/ng193

17 Duarte RV, Lambe T, Raphael JH, Eldabe S, Andronis L. Intrathecal drug delivery systems for the management of chronic noncancer pain: a systematic review of economic evaluations. Pain Pract 2018;18:666-86. doi:10.1111/ papr. 12650

18 Giovanardi CM, Cinquini M, Aguggia M. Acupuncture vs, et al. pharmacological prophylaxis of migraine: a systematic review of randomized controlled trials. Front Neurol 2020;11:576272. doi:10.3389/ fneur.2020.576272 
19 Mu J, Furlan AD, Lam WY, Hsu MY, Ning Z, Lao L. Acupuncture for chronic nonspecific low back pain. Cochrane Database Syst Rev 2020;12:CD013814

20 Reinhold T, Roll S, Willich SN, Ortiz M, Witt CM, Brinkhaus B. Cost-effectiveness for acupuncture in seasonal allergic rhinitis: economic results of the ACUSAR trial. Ann Allergy Asthma Immunol 2013;111:56-63. doi:10.1016/j. anai.2013.04.008

21 Spackman E, Richmond S, Sculpher M, et al. Cost-effectiveness analysis of acupuncture, counselling and usual care in treating patients with depression: the results of the ACUDep trial. PLoS One 2014;9:e113726. doi:10.1371/journal. pone.0113726

22 Stamuli E, Bloor K, MacPherson H, et al. Costeffectiveness of acupuncture for irritable bowel syndrome: findings from an economic evaluation conducted alongside a pragmatic randomised controlled trial in primary care. $B M C$ Gastroenterol 2012:12:149. doi:10.1186/1471 230X-12-149

23 Woods B, Manca A, Weatherly H, et al. Costeffectiveness of adjunct non-pharmacological interventions for osteoarthritis of the knee. PLOS One 2017;12:e0172749. doi:10.1371/journal. pone.0172749

24 Borah BJ, Naessens JM, Glasgow AE, Bauer BA, Chon TY. Cost-effectiveness of acupuncture in an employee population: a retrospective analysis. Complement Ther Med 2017;31:14-9. doi:10.1016/j. ctim.2017.01.002

25 Herman PM, Lavelle TA, Sorbero ME, Hurwitz EL, Coulter ID. Are nonpharmacologic interventions for chronic low back pain more cost effective than usual care? Proof of concept results from a Markov model. Spine (Phila Pa 1976) 2019;44:1456-64. doi:10.1097/BRS.0000000000003097

26 Herman PM, McBain RK, Broten N, Coulter ID. Update of Markov model on the cost-effectiveness of nonpharmacologic interventions for chronic low back pain compared to usual care. Spine
(Phila Pa 1976) 2020;45:1383-5. doi:10.1097/ BRS.0000000000003539

27 Beijing Municipal Bureau of Health, Beijing Municipal Bureau of Finance. Notice on the regulation of the publicly funded medical care of Beijing. 1990. http://www.beijing.gov.cn/zhengce/zhengcefagui/ qtwj/202008/t20200803_1969601.html

28 China National Healthcare Security Administration. Work plan for the adjustment of the national reimbursement drug list in 2021, and the application guide for the adjustment of the national reimbursement drug list in 2021. http://www.nhsa. gov.cn/art/2021/6/30/art_62_5386.html

29 Czarnawska-Iliev I, Robinson N. General practitioners' use of and attitudes to acupuncture in relation to the UK's National Institute for Health and Care Excellence (NICE) clinical guidelines-a pilot study. Eur J Integr Med 2016;8:342-5410.1016/j.eujim.2016.07.004 https://openresearch.lsbu.ac.uk/download/ f09c1f404700d8c3ac341a8b1492d187 cb181205e6e6ed262f29b91932606269/ 751670/GP\%20and\%20NICE\%20EulIM.pdf

30 Haake M, Müller HH, Schade-Brittinger C, et al. German Acupuncture Trials (GERAC) for chronic low back pain: randomized, multicenter, blinded, parallel-group trial with 3 groups. Arch Intern Med 2007;167:1892-8. doi:10.1001/ Archinte.167.17.1892

31 Scharf HP, Mansmann U, Streitberger K, et al. Acupuncture and knee osteoarthritis: a three-armed randomized trial. Ann Intern Med 2006;145:12-20. doi:10.7326/0003-4819-145-1-20060704000005

32 Witt CM, Jena S, Selim D, et al. Pragmatic randomized trial evaluating the clinical and economic effectiveness of acupuncture for chronic low back pain. Am J Epidemiol 2006;164:487-96. doi:10.1093/aje/kwi224

33 Witt CM, Reinhold T, Jena S, Brinkhaus B, Willich SN. Cost-effectiveness of acupuncture treatment in patients with headache. Cephalalgia 2008;28:334-45. doi:10.1111/ j.1468-2982.2007.01504.x
34 Reinhold T, Witt CM, Jena S, Brinkhaus B, Willich SN. Quality of life and cost-effectiveness of acupuncture treatment in patients with osteoarthritis pain. Eur I Health Econ 2008;9:209-19. doi:10.1007/s10198007-0062-5

35 Cummings M. Modellvorhaben Akupunktur-a summary of the ART, ARC and GERAC trials. Acupunct Med 2009;27:26-30. doi:10.1136/ aim.2008.000281

36 Skelly AC, Chou R, Dettori JR, et al. Noninvasive nonpharmacological treatment for chronic pain: a systematic review. US Agency for Healthcare Research and Quality, 2018. https:// effectivehealthcare.ahrq.gov/sites/default/files/pdf/ nonpharma-chronic-pain-cer-209.pdf

37 Chou R, Deyo R, Friedly J, et al. Nonpharmacologic therapies for low back pain: a systematic review for an American College of Physicians Clinical Practice Guideline. Ann Intern Med 2017;166:493-505. doi:10.7326/M16-2459

38 Database MC. Decision memo for acupuncture for chronic low back pain (CAG-00452N). Centers for Medicare \& Medicaid Services. 2020. https://www. cms.gov/medicare-coverage-database/details/ncadecision-memo.aspx?NCAld=295.

39 Drummond MF, Jefferson TO, The BMJ Economic Evaluation Working Party. Guidelines for authors and peer reviewers of economic submissions to the BMJ. BMJ 1996;313:275-83. doi:10.1136/ bmj.313.7052.275

40 Husereau D, Drummond M, Petrou S, et al, ISPOR Health Economic Evaluation Publication Guidelines-CHEERS Good Reporting Practices Task Force. Consolidated Health Economic Evaluation Reporting Standards (CHEERS)--explanation and elaboration: a report of the ISPOR Health Economic Evaluation Publication Guidelines Good Reporting Practices Task Force. Value Health 2013;16:231-50. doi:10.1016/j.jval.2013.02.002

Cite this as: $B M J$ 2022;376:e067477 http://dx.doi.org/10.1136/bmj-2021-067477 